\title{
Correction to: Primal-Dual Optimization Conditions for the Robust Sum of Functions with Applications
}

\author{
N. Dinh ${ }^{1} \cdot$ M. A. Goberna ${ }^{2} \cdot$ M. Volle ${ }^{3}$ \\ Published online: 17 December 2019 \\ ○) Springer Science+Business Media, LLC, part of Springer Nature 2019
}

\section{Correction to: \\ Applied Mathematics \& Optimization (2019) 80:643-664 https://doi.org/10.1007/s00245-019-09596-9}

The authors would like to correct the errors caused by a wrong equation $(A=B+\bar{z})$, at the end of the proof of Lemma 2.1 in the original article, which affects this lemma, two subsequent examples, as well as other statements spread along the paper.

The statement of the mentioned lemma asserts that, given a family $\left(A_{i}\right)_{i \in I}$ of convex subsets of a linear space $Z$ such that $\bigcap_{i \in I} A_{i} \neq \emptyset, A:=\bigcup_{J \in \mathcal{F}(I)} \sum_{j \in J} A_{j}$ is a convex subset of $Z$. This is not true when one takes an arbitrary $\bar{z} \in Z, \bar{z} \neq 0_{Z}$, and the sets $A_{i}=\{\bar{z}\}$ for all $i \in I$, as the set $A=\bigcup_{J \in \mathcal{F}(I)}|J|\{\bar{z}\}=\bigcup_{n \geq 1} n\{\bar{z}\}$ cannot be convex.

We now give the corrected version of this lemma and subsequent examples.

Lemma 2.1 Let $\left(A_{i}\right)_{i \in I}$ be a family of convex subsets of a linear space $Z$ such that $0_{Z} \in \bigcap_{i \in I} A_{i}$. Then $A:=\bigcup_{J \in \mathcal{F}(I)} \sum_{j \in J} A_{j}$ is a convex subset of $Z$.

Proof Notice that $\left(\sum_{j \in J} A_{j}\right)_{J \in \mathcal{F}(I)}$ is a family of convex subsets of $Z$ which is directed with respect to the inclusion. It follows that $A$ is convex.

The original article can be found online at https://doi.org/10.1007/s00245-019-09596-9.

$\bowtie \quad$ M. A. Goberna

mgoberna@ua.es

N. Dinh

ndinh02@gmail.com

M. Volle

michel.volle@univ-avignon.fr

1 International University, Vietnam National University-HCMC, Linh Trung Ward, Thu Duc District, Ho Chi Minh City, Vietnam

2 Department of Mathematics, University of Alicante, San Vicente del Raspeig, Alicante, Spain

3 Avignon University, LMA EA 2151, Avignon, France 
Example 2.1 The set $\mathcal{A}=\bigcup_{J \in \mathcal{F}(I)} \sum_{j \in J}$ epi $f_{j}^{*}$ is convex if the functions $f_{j}, j \in J$, are non-negative.

Example 2.2 The set $A:=\bigcup_{J \in \mathcal{F}(I)} \sum_{j \in J} \operatorname{dom} f_{j}^{*}$ is convex if each function $f_{j}$, $j \in J$, is bounded below.

Other corrections provoked by the above changes:

- Replace at the proof of Corollary 3.1 "Example 2.1" by "Example 2.2".

- Replace at Remark 3.4 the paragraph "If the functions ... is convex and the criteria" by "If each function $f_{i}, i \in I$, is bounded below, then (see Example 2.2) the criteria".

- Replace, at the third of the listed properties of the qualifying set $\mathcal{A}$, at the beginning of Section 5, and at the statements of Propositions 5.1-5.4, " $\bigcap_{i \in I} A_{i} \neq \emptyset$ " by " $0_{X^{*}} \in \bigcap_{i \in I} A_{i}$ " and "sup $\operatorname{supI}_{i \in I} t_{i}<+\infty\left(\operatorname{orsup}_{i \in I} t_{i} \neq+\infty\right)$ " by "sup $\operatorname{su}_{i \in I} t_{i} \leq 0$ ".

Publisher's Note Springer Nature remains neutral with regard to jurisdictional claims in published maps and institutional affiliations. 